EPJ Web of Conferences 49, 18010 (2013)

DOI: $10.1051 /$ epjconf/20134918010

(C) Owned by the authors, published by EDP Sciences, 2013

\title{
On the spin and parity of a single-produced resonance at the LHC
}

\author{
Sara Bolognesi ${ }^{1, a}$, Yanyan Gao ${ }^{2, b}$, Andrei V. Gritsan ${ }^{1, c}$, Kirill Melnikov ${ }^{1, d}$, Markus Schulze ${ }^{3, e}$, \\ Nhan V. Tran ${ }^{2, f}$, and Andrew Whitbeck ${ }^{1, g}$ \\ ${ }^{1}$ Department of Physics and Astronomy, Johns Hopkins University, Baltimore, MD 21218, USA \\ ${ }^{2}$ Fermi National Accelerator Laboratory (FNAL), Batavia, IL 60510, USA \\ ${ }^{3}$ Argonne National Laboratory (ANL), Lemont, IL 60439, USA
}

\begin{abstract}
Both LHC experiments, ATLAS and CMS, observed a new narrow resonance near $125 \mathrm{GeV}$. We demonstrate that the spin, parity, and, more generally, the tensor structure of the boson couplings of this resonance can be obtained by studying mass and angular distributions of events where the resonance decays to pairs of gauge bosons, $Z Z$, $W W$ and $\gamma \gamma$. To account for all spin correlations we calculate kinematic distributions analytically and develop a Monte Carlo generator. We also discuss how to use angular and mass distributions of the resonance decay products for optimal background rejection and for distinguishing different signal hypotheses. By the end of the $8 \mathrm{TeV}$ run of the LHC, it might be possible to separate extreme hypotheses of the spin and parity of the new resonance with a confidence level of $99 \%$ or better for a wide range of models.
\end{abstract}

\section{Introduction}

The discovery of the new boson $[1,2]$ at the LHC, further corroborated by the strong evidence from the Tevatron [3], is the culmination of the hunt for the elusive Higgs boson. Three primary decay channels $X \rightarrow Z Z, W W$, and $\gamma \gamma$ were observed experimentally. it is important to experimentally study the tensor structure of couplings of the new boson to SM fields and its $S U(2) \times U(1)$ quantum numbers (if any), avoiding theoretical prejudice. The determination of the quantum numbers of a Higgs-like particle was discussed in the literature. The strategy that we present in this talk is based on Ref. $[4,5]$. In that reference we demonstrated that $X$ decaying to two vector bosons provides an excellent channel to study the tensor structure of its couplings and outlined the general way to do so.

\footnotetext{
a e-mail: sbologne@pha.jhu.edu

be-mail:ygao@fnal.gov

ce-mail: gritsan@pha.jhu.edu

de-mail: melnikov@pha.jhu.edu

e-mail: markus.schulze@anl.gov

fe-mail: ntran@fnal.gov

ge-mail: whitbeck@pha.jhu.edu
} 

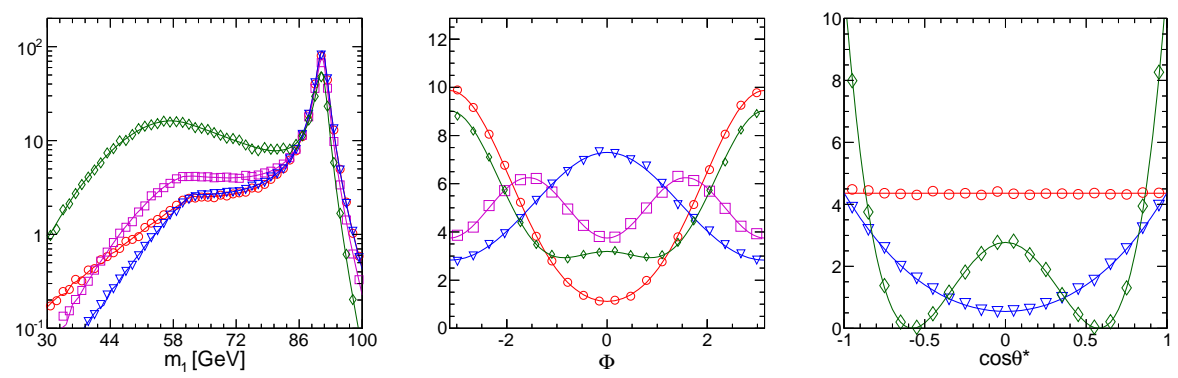

Figure 1. Distributions of some of the representative observables: $m_{1}$ in the $X \rightarrow Z Z$ analysis (left), $\Phi$ in the $X \rightarrow W W$ analysis (middle), and $\cos \theta^{*}$ in the $X \rightarrow \gamma \gamma$ analysis. Four signal hypotheses are shown: SM Higgs boson (red circles), $0^{-}$(magenta squares), $2_{m}^{+}$(blue triangles), $2_{h}^{+}$(green diamonds), as defined in Ref. [4]. Points show simulated events and lines show projections of analytical distributions. Here and throughout the paper, where only shapes of the distributions are illustrated and unless otherwise noted, units on the $y$ axis are arbitrary.

\section{$2 X \rightarrow V V$ Kinematics}

We begin by discussing kinematics of the process. Consider a sequence of processes

$$
g g / q \bar{q} \rightarrow X(q) \rightarrow V_{1}\left(q_{1}\right) V_{2}\left(q_{2}\right), \quad V_{1} \rightarrow f\left(q_{11}\right) \bar{f}\left(q_{12}\right), \quad V_{2} \rightarrow f\left(q_{21}\right) \bar{f}\left(q_{22}\right),
$$

that correspond to the production of a resonance $X$, followed by its decay to two vector bosons, followed by their decays to four fermions. The four-momenta of all particles are shown in parentheses. We denote the invariant mass of the $i$-th gauge-boson by $m_{i}^{2}=q_{i}^{2}$ and stress that it can differ from its mass $m_{V}^{2}$. We assume that the particle $X$ is produced on the mass shell, so that $q^{2}=\left(q_{1}+q_{2}\right)^{2}=m_{X}^{2}$. In what follows, we will refer to the heavier (lighter) of the two gauge bosons as $V_{1}\left(V_{2}\right), m_{1}>m_{2}$. The general scattering amplitudes that describes the interaction of the resonance with gauge bosons are given in Ref. [4]. Three invariant masses $m_{V_{1} V_{2}}, m_{1}$, and $m_{2}$, and six angles fully characterize the kinematics of the process in Eq. (1) in the rest frame of the resonance $X$. Figure 1 shows the representative observables for selected scenarios.

\section{Analysis Methods}

As a first step in understanding the spin-parity of the observed boson near $125 \mathrm{GeV}$, we perform analysis to distinguish between different hypotheses apply matrix element formalism. This is based on a simplified, but still optimal, analysis approach, that employs just two observables. Ultimately, this approach will lead to a complete multi-dimensional fit of all coupling parameters using a complete set of kinematic observables. One of the two observables is related to the resonance mass as it typically has the largest discriminating power against the background. This observable depends on the final state: four-lepton invariant mass $m_{4 \ell}$ in $X \rightarrow Z Z \rightarrow 4 \ell$, transverse mass $m_{T}$ [2] in $X \rightarrow W W \rightarrow 2 \ell 2 v$, and two-photon invariant mass $m_{\gamma \gamma}$ in $X \rightarrow \gamma \gamma$. The second observable combines other kinematic to distinguish between different signal hypotheses. In the $X \rightarrow Z Z \rightarrow 4 \ell$ analysis we build the kinematic discriminant, defined in the MELA approach adopted by the CMS experiment [2]. In the $X \rightarrow W W \rightarrow 2 \ell 2 v$ analysis, the matrix element information cannot be fully exploited due to the neutrinos in the final state. We use the most sensitive to the spin-parity of $X$ as the di-lepton invariant mass $m_{\ell \ell}$. In the $X \rightarrow \gamma \gamma$ analysis, we use the only angular variable $\cos \theta^{*}$. Figure 2 shows the distributions of the second observable for the selected signals and main background. 

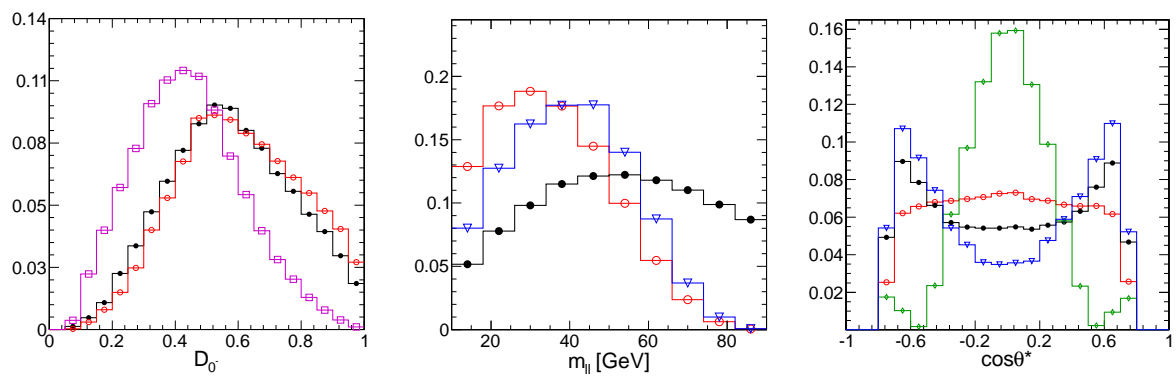

Figure 2. Signal separation observable distributions in the $Z Z$ (left), $W W$ (middle) and $\gamma \gamma$ analyses.

Table 1. Expected separation significance $\mathcal{S}\left(\right.$ Gaussian $\sigma$ ) between the SM Higgs boson $\left(0_{m}^{+}\right)$and $0^{-}$or $2_{m}^{+}$ hypotheses for the scenario corresponding to $35 \mathrm{fb}^{-1}$ of integrated luminosity at one LHC experiment.

\begin{tabular}{lccc}
\hline \hline scenario & $X \rightarrow Z Z$ & $X \rightarrow W W$ & $X \rightarrow \gamma \gamma$ \\
\hline $0_{m}^{+}$vs background & 7.1 & 4.5 & 5.2 \\
\hline $0_{m}^{+}$vs $0^{-}$ & 4.1 & 1.1 & 0.0 \\
$0_{m}^{+}$vs $2_{m}^{+}$ & 2.2 & 2.5 & 2.5 \\
\hline \hline
\end{tabular}

We use an extended maximum-likelihood fit [4] to extract simultaneously the signal and background yields. The likelihood is defined as

$$
\mathcal{L}_{k}=\exp \left(-n_{\mathrm{sig}}-n_{\mathrm{bkg}}\right) \prod_{i}\left(n_{\mathrm{sig}} \times \mathcal{P}_{\mathrm{sig}}^{k}\left(\vec{x}_{i} ; \vec{\alpha} ; \vec{\beta}\right)+n_{\mathrm{bkg}} \times \mathcal{P}_{\mathrm{bkg}}\left(\vec{x}_{i} ; \vec{\beta}\right)\right),
$$

where $n_{\text {sig }}$ is the number of signal events, $n_{\mathrm{bkg}}$ is the number of background events and $\mathcal{P}\left(\vec{x}_{i} ; \vec{\alpha} ; \vec{\beta}\right)$ is the probability density function for background or signal for different spin hypotheses, $k$. Each event candidate, $i$, is characterized by a set of two observables $\vec{x}_{i}=(m, D)$. The signal coupling parameters are collectively denoted by $\vec{\alpha}$, and the remaining parameters by $\vec{\beta}$. The correlated $(m, D)$ distribution is parameterized with a binned histogram (template) using simulation. The likelihood $\mathcal{L}_{k}$ in Eq. (2) is evaluated independently for each spin hypothesis $k$. The likelihood ratio between the SM Higgs boson and other hypothesis is then used to quantify the separation as described in Ref. [5].

\section{Results}

In Table 1 we show examples of hypothesis separation expectations, per each LHC experiment, by the end of the $8 \mathrm{TeV}$ LHC run, assuming $35 \mathrm{fb}^{-1}$ of integrated luminosity.

\section{References}

[1] ATLAS collaboration, Phys. Lett. B 716, 1 (2012),

[2] CMS collaboration, Phys. Lett. B 716, 30 (2012),

[3] CDF and D0 collaborations, Phys. Rev. Lett. 109, 071804 (2012).

[4] Y.Y. Gao et al., Phys. Rev. D 81, 075022 (2010).

[5] S. Bolognesi et al., Phys. Rev. D 86, 095031 (2012). 